\title{
Anthropometric and glucometabolic changes in an aged mouse model of lipocalin-2 overexpression
}

\author{
Principi $\mathrm{E}^{1}$, Buschiazzo $\mathrm{A}^{2}$, Papait $\mathrm{A}^{1}$, Castagnola $\mathrm{P}^{3}$, Costa $\mathrm{D}^{1}$, Scussolini $\mathrm{M}^{4}$, Marini $\mathrm{C}^{5}$, \\ Sambuceti $\mathrm{G}^{2}$, Strollo $\mathrm{F}^{6}$ and Tavella $\mathrm{S}^{1}$
}

1- Dipartimento di Medicina Sperimentale, Università degli Studi di Genova \& Ospedale Policlinico San Martino, Largo Rosanna Benzi 10, 16132 Genova, Italia.

2- Dipartimento di Scienze della Salute, Università degli Studi di Genova \& Ospedale Policlinico San Martino, Genova, Italia.

3- RCCS AOU San Martino-IST, Istituto Nazionale per la Ricerca sul Cancro, Genova, Italia.

4- Dipartimento di Matematica, Università degli Studi di Genova, Italia.

5- CNR Istituto di Bioimmagini e Fisiologia Molecolare, Milano, Italia.

6- Dipartimento di Scienze Farmacologiche e Biomolecolari, Università degli Studi di Milano, Italia

\author{
Corresponding author: \\ Sara Tavella $\mathrm{PhD}$ \\ Dipartimento di Medicina Sperimentale \\ \& Terapie Oncologiche Integrate \\ Università di Genova \\ \& Ospedale Policlinico San Martino \\ L.go R. Benzi 10 \\ 16131 Genova, Italia \\ phone: +390105558241 \\ fax: +390105558257 \\ email: sara.tavella@unige.it
}

The authors declare no conflict of interests

Running title: Adipose tissue changes in LCN2 overexpressing mice

KEY WORDS: ageing, lipocalin-2, glucose metabolism, adipose tissue 


\section{ABSTRACT}

Background: Lipocalin-2 (LCN2) is widely expressed in the organism with pleiotropic roles.

In particular its over-expression correlates with tissue stress conditions including inflammation, metabolic disorders, chronic diseases and cancer.

Objectives: assess the effects of systemic LCN2 overexpression on adipose tissue and glucose metabolism.

Subjects: Transgenic mouse model with systemic LCN2 overexpression (LCN2-Tg) and age/sex matched wild type control mice.

Methods: Metabolic cages and body mass parameters evaluation; adipose tissue histology and Real-Time PCR analysis; glucose tolerance test; cytofluorimetric, micro-PET and serum analysis.

Results: LCN2-Tg mice were smaller compared to controls but they ate $(\mathrm{P}=0.0156)$ and drank $(\mathrm{P}=$ 0.0057) more. Aged LCN2-Tg mice displayed a lower body mass index $(\mathrm{P}=0.0018)$ and a higher amount of visceral adipose tissue. Furthermore, LCN2-Tg mice with body weight $\geq 20 \mathrm{~g}$, showed adipocytes with a higher cell area $(\mathrm{P}<0.0001)$. LCN2-Tg also showed an altered expression of genes involved in adipocyte differentiation, obese phenotype and inflammation. In particular, mRNA levels of adipocyte-derived PPAR $\gamma(\mathrm{P}=<0.0001)$, SRBF1 ( $\mathrm{P}<0.0001)$, FBP4 $(\mathrm{P}=0.056), \mathrm{TNF} \alpha$ $(\mathrm{P}=0.0391)$, IL6 $(\mathrm{P}=0.0198)$ and leptin $(\mathrm{P}=0.0003)$ were all increased. Furthermore, glucose tolerance test in LCN2-Tg mice showed a delay in glucose tissue uptake from blood to tissues (30 minutes $\mathrm{P}=0.032,60$ minutes, $\mathrm{P}=0.0171$ ) and micro-PET analysis demonstrated reduced whole body glucose consumption along with a decreased MRGlu (metabolic rate of glucose) in both muscle and brown adipose tissue $(\mathrm{P}=0.0247)$. Nevertheless, an almost nine-fold acceleration of hexokinase activity was observed in LCN2-Tg mice liver compared to the controls $(\mathrm{P}=0.0027)$. Moreover, AST and $\operatorname{ALT}(\mathrm{P}=0.0421 ; \mathrm{P}=0.0403)$ were increased confirming a liver involvement.

Conclusions: We show that LCN2 profoundly impacts adipose tissue size and function and glucose metabolism indicating that LCN2 should be considered as a risk factor for metabolic disorders leading to obesity 


\section{INTRODUCTION}

Lipocalin-2 belongs to a superfamily of more than 20 proteins identified originally in human neutrophil granules ${ }^{1}$. Lipocalins bind a wide array of small hydrophobic ligands, which include bacterial siderophores. LCN2 is involved in innate immunity and regulates several cellular processes ${ }^{2}$. It is expressed in several tissues and overexpressed during tissue stress conditions like postpartum uterus involution ${ }^{3,4}$. Within the skeleton, LCN2 is expressed by chondrocytes in the hypertrophic cartilage of the growth plate of long bones, that undergo endochondral ossification ${ }^{5}$, and by differentiating osteoblasts along with its receptor ${ }^{6}$.

LCN2 is widely overexpressed under pathological conditions such as liver and lung inflammation ${ }^{4,7}$ where macrophages and cells of the immune response produce a large amount of this protein, which correlates with cirrhosis-dependent liver failure ${ }^{8,9}$. Moreover, LCN2 overexpression in the organism actively participates to several disorders and diseases. In bone LCN2 can unbalance, when overexpressed in mice, the finely tuned bone turnover and modify the bone marrow environment ${ }^{10}$. In chronic kidney disease, LCN2 overexpression seems to play an active role in causing the renal phenotype ${ }^{11}$. In ovarian ${ }^{12}$ pancreatic ${ }^{13}$ and breast cancers ${ }^{14}$ LCN2 is an important pro-survival protein.

Among the pathological conditions in humans, obesity has increased at an alarming rate in the last twenty years, thus becoming a major health and economic issue for industrialized countries, mainly due to its high number of complicating diseases ${ }^{15}$. For this reason, clinical interest on adipose tissue has been rapidly developing and, thanks to recent studies, this tissue is no longer believed to have uniquely a lipid-storage capability but it is recognized as a diffused endocrine organ meant at producing hormones and cytokines ${ }^{16}$. The latter, when found within adipocytes, are also termed adipokines and play a dramatic role in the modulation of the adipose tissue response, linking obesity to inflammation. An inflammatory state is associated with the current pandemic of obesity and it is an important pathogenetic factor that contributes to the increased prevalence of the so-called metabolic syndrome ${ }^{17,18}$. Nowadays it is a common opinion that LCN2 is endowed with proinflammatory and metabolism-disrupting properties ${ }^{19}$. Furthermore, recent studies suggest that this protein is an independent risk factor for both inflammation and insulin resistance before diabetes onset. Overall, LCN2 is recognized as one of the adipokines playing a role in obesity and metabolic syndrome ${ }^{20}$.

Although recent studies reported a correlation between serum LCN2 concentration, glucose metabolism alterations and inflammatory markers ${ }^{18}$ the underlying mechanisms remain largely undefined. In particular, ablation of LCN2 expression has been found to only marginally improve obesity-associated glucose intolerance without affecting either age- or obesity-mediated insulin 
resistance ${ }^{20}$. Moreover, a recent report suggested that bone-derived LCN2 regulates metabolism by suppressing appetite through MC4R in the hypothalamus, thus assigning a leading role to LCN2 in bone endocrine function ${ }^{21}$.

In this scenario a better knowledge about LCN2 effects at the organism level is crucial for our understanding of the pathogenesis of metabolic disorders and possibly to open up new paths for therapeutic approaches.

In the present study, we took advantage of a transgenic mouse model over-expressing LCN2 under the control of the mouse pro alpha 1 type I collagen promoter, which was previously generated in our laboratory ${ }^{22}$. In this mouse model LCN2 is overexpressed by osteoblasts and released in the serum and therefore this model is suitable to study LCN2 pleiotropic role in the body.

In 18 month old LCN2-Tg mice we observed an increased expression of some relevant genes linked to obesity such as leptin, FBP4, TNF $\alpha$ and IL-6 along with an altered glucose tolerance. Moreover, LCN2 overexpression promoted glucose metabolism changes and was associated to a large increase in adipose tissue volume, body mass, food and water intake. These data point to LCN2 altered expression as a promoting factor for the development of metabolic disorders in ageing.

\section{MATERIAL AND METHODS}

\section{Animal maintenance and generation of LCN2-Tg Mice}

Procedures involving the animals and their care were all performed according to the institutional guidelines, in compliance with national and international laws and guidelines for the use of animals in biomedical research. Mice were bred and maintained at the Institution's animal facility (IRCCS AOU - San Martino - IST, Genova, Italia). LCN2-Tg mice were generated as previously described

${ }^{22}$. Briefly, the full-length cDNA coding for mouse LCN2 was cloned between the mouse pro $\alpha 1$ collagen I promoter ${ }^{23}$ and the Bovine growth hormone polyadenylation (bpA) plasmid sites. Pronuclei of FVB mice (Charles River) fertilized eggs were injected with this recombinant construct and then implanted in pseudo-pregnant foster mothers. Transgenic mice were identified by PCR analysis of tail genomic DNA with LCN2 cDNA and bpA specific primers.

All analyses were performed on 18 month old mice.

\section{Metabolic analysis}


Food and water consumption were measured in 10 control Wt and $11 \mathrm{LCN} 2-\mathrm{Tg}$ mice that were weighed and housed for 24 hours in metabolic cages. For both groups, body surface area was derived from the DuBois equation:

$$
\text { Body surface }\left(\mathrm{m}^{2}\right)=0.007184 \mathrm{X} \text { weight }\left(\mathrm{Kg}^{0.425}\right) \mathrm{X} \text { height }\left(\mathrm{cm}^{0.725}\right)
$$

Thereafter, body mass index (BMI) was calculated as the ratio between body weight and surface $\operatorname{area}\left(\mathrm{g} / \mathrm{m}^{2}\right){ }^{24}$.

\section{Histology}

For standard histology, visceral fat was removed, weighed, and fixed immediately in $4 \%$ buffered formaldehyde (Sigma Aldrich). After dehydration in a progressive series of ethanol, fat samples were clarified in xylene and paraffin embedded. Five $\mu \mathrm{m}$ sections were obtained and stained with Haematoxylin and Eosin (H\&E) for histological examination. At least 10 slides for each sample were scored for fat histology an at least 5 tissue sections were analysed for each animal. Images were acquired by an Axiovert 200M (Zeiss, Germany). Adipocytes were counted in fat sections from Wt and LCN2-Tg mice and surface area was measured using an image analysis software (ImageJ, NIH USA). Each count represents the mean value of 5 different blinded fields.

\section{Adipose macrophage immune profiling}

After 8 hours of starvation mice were sacrificed and visceral fat was dissected, minced into fine pieces $\left(2-6 \mathrm{~mm}^{3}\right)$ and digested in a buffer isolation solution (PBS w/o Ca2+ $\mathrm{Mg} 2+, 1.5 \% \mathrm{FBS}$, Collagenase I $1 \mathrm{mg} / \mathrm{ml}$ ). The homogenized suspensions were maintained in water bath at $37^{\circ} \mathrm{C}$ with intermittent shaking for 1.5 hours, then passed through a $7 \mu \mathrm{m}$ mesh. The cells suspension with the was centrifuged at $400 \mathrm{~g}$ for 10 minutes at $25^{\circ} \mathrm{C}$ and then washed in RPMI1640. The following antibodies were used to discriminate two different subsets of macrophages (MФ): CD45 APC, F4/80 PeCy7, CD86 FITC for the M1 inflammatory MФ, and the CD206 FITC for the M2 antiinflammatory МФ. Due to the overlap of the specific MФ subset markers (CD86 and CD206), after accurate suspension, each sample was equally divided in two separate tubes. Incubations were performed at saturating antibody concentrations for $30 \mathrm{~min}$ at $4^{\circ} \mathrm{C}$. For each sample $10^{5}$ cells were 
run on a FACS CyAN ADP flow cytometer (Beckman-Coulter, Brea, California). Results were analysed with the Summit software (Beckman-Coulter).

\section{Real time PCR analysis}

Small visceral adipose tissue samples from $12 \mathrm{LCN} 2-\mathrm{Tg}$ and $8 \mathrm{Wt} 18$ months old mice were isolated and homogenized in Qiazol reagent (Qiagen). Subsequently, samples were extracted by Rneasy plus mini kit (Qiagen), quantified by optical density (OD) measurement, and checked for quality. cDNA synthesis was performed using Omniscript Reverse Transcription Kit (Qiagen). Real time PCR reaction was performed using specific primers for each gene (Table 1) and Platinum SYBR green qPCR SuperMix-UDGwith ROX kit (Invitrogen). Detection was performed using the 7500 fast real time PCR system (Thermo Fisher Scientific). Relative expression values with standard errors were obtained using the Thermo Fisher Scientific software and normalized to the expression of the housekeeping gene Hprt. Each sample was run in triplicate.

\section{Glucose tolerance test}

After a short fasting time (5-6 hours) $8 \mathrm{Wt}$ and $9 \mathrm{LCN} 2-\mathrm{Tg}$ mice were intraperitoneally injected with a glucose solution $(1,5 \mathrm{mg} / \mathrm{g})$. Glucose concentrations were measured before the glucose administration and monitored every 10 to 30 minutes for 30 minutes to 2 hours after the injection ${ }^{25}$.

\section{micro-PET analysis}

In vivo imaging was performed according to a protocol validated in our lab 262728 . Daily quality controls always documented a radiochemical $\geq 98 \%$ purity of in house produced 18Ffluorodeoxyglucose (FDG).

Before PET scan, $7 \mathrm{Wt}$ and $8 \mathrm{LCN}-\mathrm{Tg}$ mice were kept under fasting conditions with free access to water for 6 hours. Mice were weighted and anesthesia was induced by intra-peritoneal administration of ketamine $(100 \mathrm{mg} / \mathrm{kg})$ and xylazine $(10 \mathrm{mg} / \mathrm{kg})$ (Imalgere 1000, Bio98 Srl, Milan, Italy, respectively). Serum glucose level was tested and animals were positioned on the bed of a dedicated micro-PET system, (Albira, Bruker, Billerica, MA, USA), the double-ring configuration of which permitted to cover the whole animal body in a single bed position. A dose of 3-4 MBq of FDG was then injected through a tail vein, soon after start of a list mode acquisition lasting 50 minutes.

The whole dataset was thus binned using the following framing rate: $10 \times 15 \mathrm{~s}, 5 \times 30 \mathrm{~s}, 2 \times 150 \mathrm{~s}$, 6x300 s, 1 x600 s. PET data were reconstructed using a maximal likelihood expectation 
maximization method (MLEM). An experienced observer, kept unaware of the experimental type of the analyzed model, identified a volume of interest (VOI) in the left ventricle. Then, the computer was asked to plot the time-concentration curve within this VOI throughout the whole acquisition to define arterial tracer input function. Whole body FDG clearance (in $\mathrm{ml} x \mathrm{~min}-1$ ) was calculated, using the conventional stochastic approach, as the ratio between injected dose and integral of input function from 0 to infinity, fitting the last 20 minutes with a mono-exponential function ${ }^{29}$. This value was thus multiplied by serum glucose level to measure whole body glucose consumption and then normalized based on body weight and thus expressed as $\mu \mathrm{M} \times \min ^{-1} \times 100 \mathrm{~g}^{-1}$. All dynamic scans were processed according to the Gjedde-Patlak ${ }^{30}$ graphical approach to compartmental analysis by using the routine of a dedicated software (PMOD, Zurich, Switzerland). Briefly, the software utilizes the input function and transforms the original tissue activity measurements by fitting the data in each voxel with the slope of the regression line defined by the model. In all cases, the lumped constant value was set at 1. Further VOIs were drawn on the obtained parametric maps to estimate metabolic rate of glucose (MRGlu) in brown adipose tissue (BAT) and in hind limb skeletal muscles (SM).

Hepatic FDG kinetics was assessed according to a method previously validated in our lab ${ }^{29}$. Briefly, the algorithm utilizes time-concentration curve in the gut to derive portal vein input function and provides a description of parameters quantifying phosphorylation rate ( $\mathrm{kmf}$ ) and dephosphorylation rate $(\mathrm{kfm})$ of FDG within hepatocytes.

\section{Serum Analysis}

All animals were sacrificed after a 6 hours of fasting to allow cardiac blood samples collection. Samples were centrifuged for 60 minutes at $7,500 \times \mathrm{g}$ and then stored at $-80^{\circ} \mathrm{C}$. In addition to glucose, urea, creatinine, triglycerides, total bilirubin, bile acids, AST, ALT were also assayed in plasma (all Mindray reagents) using a clinical-standard automatic chemistry analyser (BS-380 Mindray).

\section{Statistical analysis}

All experiments were performed using at least $7 \mathrm{Wt}$ and $7 \mathrm{LCN} 2-\mathrm{Tg} 18$ month old mice. Data were expressed as mean \pm SEM and analyzed using GraphPad Prism software (GraphPad Software, Inc., La Jolla, CA). Statistical analysis was performed by a two-tailed t-test.

\section{RESULTS}




\section{LCN2-Tg mice weight and metabolism}

To investigate the role of LCN2 in the control of body mass, fat accumulation in the adipose tissue and food and water intake, we used a transgenic mouse model that overexpresses LCN2 in the serum $^{22}$. As previously reported ${ }^{6}$, we found that 18 month old LCN2-Tg displayed a smaller body size and weight compared to Wt controls (Fig 1A). Similarly, the body mass index (BMI) of 18 month old mice was lower for LCN2-Tg compared to Wt $(\mathrm{P}=0.0018)$ (Fig. 1B). Furthermore, LCN2-Tg mice showed a higher amount of fat per body weight (Fig 1C, D). To assess the levels of the LCN2 mRNA in the adipose tissue, we performed a real-time PCR analysis. This analysis showed that the LCN2 mRNA was expressed at much higher levels in the adipose tissue of LCN2Tg mice compared to $\mathrm{Wt}(\mathrm{P}<0.001)$ (Fig 1E). Despite their lower body mass and size, LCN2-Tg showed a higher food $(\mathrm{P}=0.0156)$ and water $(\mathrm{P}=0.0057)$ intake (Fig.1F).

\section{Physiology and inflammatory status of LCN2-Tg mice visceral adipose tissue}

To better characterize the increased visceral adipose tissue, we investigated this issue at a histological and molecular level (Fig. 2). Necroscopy on mice confirmed a higher amount of visceral adipose tissue in LCN2-Tg (Fig. 2A) and related H\&E staining showed no major leucocytes infiltration. However, this analysis revealed in the adipose tissue of LCN2-Tg with a body weight higher than $20 \mathrm{~g}$, a lower total number of adipocyte compared to $\mathrm{Wt}$ controls $(\mathrm{P}=$ 0.0001) (Fig. 2B). Furthermore, LCN2-Tg adipocytes showed a higher degree of hypertrophy because we detected a larger mean cellular area $(\mathrm{P}<0.0001)$ (Fig. 2C)

From these results we hypothesized an aberrant expression of genes involved in adipocyte differentiation, inflammation and body weight. Therefore, we performed a real-time PCR analysis on adipose tissue in randomly selected Wt and LCN2-Tg mice.

In particular, we evaluated mRNA levels for PPAR $\gamma$ and SRBF1, two relevant proteins expressed during adipocyte differentiation ${ }^{31}$ and the mRNA levels for the FBP4 (fatty acid binding protein) and Leptin, which are involved in both obesity and metabolic syndrome ${ }^{32}$. Fig. 2D and Fig. 2E show that these mRNAs were all up-regulated $(\mathrm{P}<0.001 ; \mathrm{P}<0.001 ; \mathrm{P}=0.0056 ; \mathrm{P}=0.0003)$ in LCN2Tg adipose tissue compared to $\mathrm{Wt}$.

Since obesity often correlates with increased circulating inflammatory adipokines we studied by real-time PCR analysis the expression of the pro-inflammatory cytokine e.g. TNF $\alpha$ and IL-6 mRNAs in the adipose tissue of randomly selected mice. This analysis revealed a significantly increased expression of both TNF $\alpha(\mathrm{P}=0.0391)$ and IL-6 transcripts $(\mathrm{P}=0.0198)$ in LCN2-Tg mice (Fig. 2E) compared to controls. 
Fat resident macrophages are major players in the modulation of the inflammatory microenvironment within the adipose tissue ${ }^{33}$. To assess whether higher levels of LCN2 affects the presence of these cells in the adipose tissue, we measured by FACS analysis the percentage of inflammatory M1 and anti-inflammatory M2 macrophages, identified by the expression of CD86 and CD206, respectively. This analysis showed similar percentage of fat resident M1 and M2 macrophages in LCN2-Tg and Wt mice (Fig. 2F)

\section{Glucose homeostasis in LCN2-Tg mice}

Given the alterations found in the LCN2-Tg mice adipose tissue, we hypothesized the presence of alterations in glucose homeostasis in these mice. Therefore we performed a glucose tolerance test (GTT), which showed no differences between the Wt and LCN2-Tg mice in term of starting and final values of glucose concentration (Fig. 3). However, a relative delay in glucose downward slope occurred in LCN2-Tg mice, with significantly higher glucose levels attained at $30 \mathrm{~min}(\mathrm{P}=0.032)$ and $60 \min (\mathrm{P}=0.0171)$ after intraperitoneal injection.

\section{Micro-PET}

The glucose tolerance test suggested a possible slower glucose transfer from blood to tissues in LCN2-Tg compared to Wt mice. To test this hypothesis, we performed in vivo total body microPET analysis. LCN2-Tg mice started with lower glucose serum levels ( $\mathrm{P}=0.0499)$ (Fig. 4A). During micro-PET analysis their glucose disposal was reduced with respect to Wt (Fig. 4B, C). Similarly, transgenic mice attained lower MRGlu values both in muscle (Fig. 4D), and in brown adipose tissue $(\mathrm{P}=0.0247)$ (Fig. 4E). Altogether, these data suggest that LCN2 overexpression significantly impaired tissue glucose utilization. This behaviour was not confirmed by fasting serum glucose level that, in fact, was lower in LCN2-Tg than in Wt. The mismatch between lower sugar utilization and reduced glucose availability was explained by the effect of LCN2 overexpression on liver metabolism which, under fasting conditions, showed an almost nine-fold acceleration of hexokinase activity with respect to $\mathrm{Wt}$ mice as documented by $\mathrm{k}_{\mathrm{mf}}(\mathrm{P}=0.0027)$ (Fig. 4F). Under this condition, LCN2-Tg liver buffer effect on serum glucose was relatively hampered by a less prominent glucose G6P-phosphatase dependent dephosphorylation process, which, however, did not reach statistical significance (Fig. 4G).

\section{Serum analysis}

Glucose homeostasis alteration and visceral adipose tissue accumulation is closely associated to 
obesity ${ }^{32}$. This disease is characterized by dyslipidemia and a low inflammatory state that in humans is often associated with other serum parameter alterations. In our LCN2-Tg mice however total cholesterol was significantly lower $(\mathrm{P}=0.0193)$ (Fig.5A), while triglyceride levels tended to be lower in comparison to $\mathrm{Wt}$, without reaching statistical significance (Fig.5A). We could not find any relevant differences in serum of creatinine (Fig. 5B), bile acids (Fig. 5C), or in total bilirubin levels between the two mice groups (Fig. 5D). By contrast, in LCN2-Tg mice we found increased serum urea $(\mathrm{P}=0.0025)$ (Fig. 5E), aspartate aminotransferase (AST) and alanine aminotransferase (ALT) levels ( $\mathrm{P}=0.0421)$ (Fig. 5F).

\section{DISCUSSION}

Lipocalin-2 is expressed in several tissues and its overexpression is linked to several pathological outcomes. However, the exact molecular mechanisms at the basis of the LCN2 effects in different tissue and organs are not clear yet. LCN2-Tg mice, which was generated in our laboratory, overexpressed LCN2 within blood and tissues ${ }^{6}$, making this mouse model a good tool to investigate the effects of the up-regulation of LCN2 expression in the whole organism. Previous works from our group assessed the effects of persistent LCN2 overexpression on bone physiology at first ${ }^{6}{ }^{34}$. In particular we reported bone loss and bone marrow modifications, including hematopoietic changes. Here we show that, in aged mice, persistent LCN2 overexpression interferes with body weight and glucose metabolism.

In particular, LCN2-Tg mice displayed higher amount of visceral adipose tissue and those mice heavier than $20 \mathrm{~g}$ presented bigger adipocytes than controls. This phenotype is accompanied by an increase of the BMI. However, further studies are needed to understand the higher variability in body weight and BMI displayed by LCN2-Tg compared to age-matched Wt mice. Several evidences show that obesity is characterized by enlarged adipose tissue with increased production of both LCN2 and other pro-inflammatory adipokines, including TNFa, IL-6, MCP-1, and Resistin

${ }^{35}$ which are strongly linked to metabolic abnormalities ${ }^{32}$. Our gene expression analysis, performed on adipose tissue of randomly selected LCN2-Tg mice, showed that besides the expected overexpression of LCN2 mRNA, the transcripts for two pro-inflammatory adipokines, TNFa and IL-6 are up-regulated in these mice compared to Wt.

These data show that the adipose tissue in LCN2-Tg mice developed an inflammatory obese-like phenotype. However, as we could not detect by histology and FACS analyses neither a relevant infiltration by immune cells or changes in the ratio between M2 pro-inflammatory and M1 anti- 
inflammatory macrophages, we hypothesize that LCN2 exerts its pro-inflammatory effects mainly through adipocytes. Expression analysis also revealed that, PPAR $\gamma, \mathrm{SRBF} 1$, leptin and FBP4 mRNAs were all up-regulated in LCN2-Tg adipose tissue. Leptin is a hormone, which contributes to energy balance regulation by inhibiting hunger ${ }^{36}$ through its hypothalamic arcuate nucleus receptors ${ }^{37}$. In obesity, a decreased sensitivity to leptin occurs ${ }^{38}$ along with a positive correlation between obesity and high systemic leptin levels ${ }^{39}$. Our data, in view of these reports, strongly suggests the existence of leptin resistance in Lcn2-Tg mice. In LCN2-Tg mice, indeed, both food and water intake are higher compared to Wt mice, despite a smaller body size was observed in the former. Furthermore, the increase in PPAR $\gamma$, and SRBF1 mRNAs strongly suggest a high metabolic activity of fat tissue in LCN2-Tg as these genes are very active during adipocyte differentiation and up-regulated in cardiovascular and metabolic abnormalities 404142 .

Obesity has effects on glucose tolerance, which is often decreased in this condition. A slightly improved glucose tolerance was found in high-fat diet fed mice with LCN2 deficiency without any differences in insulin sensitivity ${ }^{20}$. A different study suggested that, compared to Wt mice, LCN2 deficient animals had deleterious diet effects, including dyslipidaemia, fatty liver disease, insulin resistance and lower mitochondrial oxidative capacity ${ }^{43}$. In our mice overexpressing LCN2, the GTT curve showed that LCN2-Tg mice took longer to transfer glucose from blood to tissues compared to Wt. Our micro-PET data revealed an unexpected near-nine-fold acceleration of hexokinase activity in LCN2-Tg with respect to Wt mice as documented by $\mathrm{K}_{\mathrm{mf}}$. It is well known that liver plays a crucial role in glucose systemic level changes ${ }^{20}$. Therefore, micro-PET data together with the observed alterations in circulating liver function markers warrant further investigations upon hepatic involvement in LCN2-Tg mice. Interestingly, a previous study reported that LCN2 is a major factor leading to metabolic liver alterations ${ }^{42}$. Moreover, before micro-PET analysis glucose levels were tested in LCN2-Tg mice: this revealed that in this case the starting point was different in $\mathrm{Wt}$ compared to LCN2-Tg who displayed slightly lower glucose concentrations. However, under vivarium conditions, glucose levels did not significantly differ between LCN2-Tg and Wt groups. Such discrepancy might be explained by the stress typically faced by the animals during transportation and before undergoing micro-PET. Should this be the case, however, LCN2-Tg mice could be thought to react to stress differently from Wt mice.

It should also be taken into account that the metabolic alteration observed in LCN2-Tg may also due in part to the skeleton, which is an endocrine organ per se and that osteoblasts contribute to energy metabolism through the expression of their own endocrine signals, including osteocalcin, a peptide involved in insulin secretion/sensitivity and energy expenditure regulation ${ }^{44}$. In particular, 
we previously observed in LCN2-Tg mice bone a decreased amounts of osteocalcin-related mRNA and this could contribute to the observed changes in glucose metabolism ${ }^{6}$.

In conclusion, here we show that LCN2 profoundly impacts adipose tissue size and function and glucose metabolism indicating that LCN2 should be considered as a risk factor for metabolic disorders leading to obesity.

\section{REFERENCES}

1 Flo TH, Smith KD, Sato S, Rodriguez DJ, Holmes MA, Strong RK et al. Lipocalin 2 mediates an innate immune response to bacterial infection by sequestrating iron. 2004; : 917 921.

2 Flower DR. The lipocalin protein family : structure and function. 1996; 14: 1-14.

3 Wu G, Li H, Zhou M, Fang Q, Bao Y, Xu A et al. Mechanism and clinical evidence of lipocalin-2 and adipocyte fatty acid-binding protein linking obesity and atherosclerosis. Diabetes. Metab. Res. Rev. 2014; 30: 447-456.

4 Liu Q, Ryon J, Nilsen-Hamilton M. Uterocalin: A mouse acute phase protein expressed in the uterus around birth. Mol Reprod Dev 1997; 46: 507-514.

5 Cermelli S, Zerega B, Carlevaro M, Gentili C, Thorp B, Farquharson C et al. Extracellular fatty acid binding protein (Ex-FABP) modulation by inflammatory agents: 'physiological' acute phase response in endochondral bone formation. Eur J Cell Biol 2000; 79: 155-164.

6 Costa D, Lazzarini E, Canciani B, Giuliani A, Spanò R, Marozzi K et al. Altered bone development and turnover in transgenic mice over-expressing Lipocalin-2 in bone. J Cell Physiol 2013; 228: 2210-2221.

7 Liu Q, Nilsen-Hamilton M. Identification of a new acute phase protein. J Biol Chem 1995; 270: $22565-70$.

8 Meheus LA, Fransen LM, Raymackers JG, Blockx HA, Van Beeumen JJ, Van Bun SM et al. Identification by microsequencing of lipopolysaccharide-induced proteins secreted by mouse macrophages. J Immunol 1993; 151: 1535-1547.

9 Ariza X, Graupera I, Coll M, Sol?? E, Barreto R, Garc??a E et al. Neutrophil gelatinaseassociated lipocalin is a biomarker of acute-on-chronic liver failure and prognosis in cirrhosis. J Hepatol 2016; 65: 57-65.

10 Costa D, Principi E, Lazzarini E, Descalzi F, Cancedda R, Castagnola P et al. LCN2 overexpression in bone enhances the hematopoietic compartment via modulation of the bone marrow microenvironment. J Cell Physiol 2017. doi:10.1002/jcp.25755.

11 Viau A, El Karoui K, Laouari D, Burtin M, Nguyen C, Mori K et al. Lipocalin 2 is essential for chronic kidney disease progression in mice and humans. J Clin Invest 2010; 120: 40654076.

12 Bartsch S, Tschesche H. Cloning and expression of human neutrophil lipocalin cDNA derived from bone marrow and ovarian cancer cells. FEBS Lett 1995; 357: 255-9.

13 Furutani M, Arii S, Mizumoto M, Kato M, Imamura M. Identification of a neutrophil 
gelatinase-associated lipocalin mRNA in human pancreatic cancers using a modified signal sequence trap method. Cancer Lett 1998; 122: 209-214.

14 Stoesz SP, Friedl A, Haag JD, Lindstrom MJ, Clark GM, Gould MN. Heterogeneous expression of the lipocalin NGAL in primary breast cancers. Int J Cancer 1998; 79: 565-572.

15 Auguet T, Quintero Y, Terra X, Martinez S, Lucas A, Pellitero S et al. Upregulation of lipocalin 2 in adipose tissues of severely obese women: positive relationship with proinflammatory cytokines. Obes (Silver Spring) 2011; 19: 2295-2300.

16 Trayhurn P, Wood IS. Adipokines: inflammation and the pleiotropic role of white adipose tissue. Br J Nutr 2004; 92: 347.

17 Jang Y, Lee JH, Wang Y, Sweeney G. Emerging clinical and experimental evidence for the role of lipocalin-2 in metabolic syndrome. Clin Exp Pharmacol Physiol 2012; 39: 194-9.

18 Moreno-Navarrete JM, Manco M, Ibáñez J, García-Fuentes E, Ortega F, Gorostiaga E et al. Metabolic endotoxemia and saturated fat contribute to circulating NGAL concentrations in subjects with insulin resistance. Int J Obes (Lond) 2010; 34: 240-9.

19 Tan BK, Adya R, Shan X, Syed F, Lewandowski KC, O'hare JP et al. Ex vivo and in vivo regulation of lipocalin-2, a novel adipokine, by Insulin. Diabetes Care 2009; 32: 129-131.

20 Jun LS, Siddall CP, Rosen ED. A minor role for lipocalin 2 in high-fat diet-induced glucose intolerance. Am J Physiol Endocrinol Metab 2011; 301: E825-35.

21 Mosialou I, Shikhel S, Liu J-M, Maurizi A, Luo N, He Z et al. MC4R-dependent suppression of appetite by bone-derived lipocalin 2. Nature 2017; 543: 385-390.

22 Costa D, Biticchi R, Negrini S, Tasso R, Cancedda R, Descalzi F et al. Lipocalin-2 controls the expression of SDF-1 and the number of responsive cells in bone. Cytokine 2010; 51: 4752.

23 Rossert J a, Chen SS, Eberspaecher H, Smith CN, de Crombrugghe B. Identification of a minimal sequence of the mouse pro-alpha 1(I) collagen promoter that confers high-level osteoblast expression in transgenic mice and that binds a protein selectively present in osteoblasts. Proc Natl Acad Sci U S A 1996; 93: 1027-1031.

24 Gargiulo S, Gramanzini M, Megna R, Greco A, Albanese S, Manfredi C et al. Evaluation of growth patterns and body composition in $\mathrm{c} 57 \mathrm{bl} / 6 \mathrm{j}$ mice using dual energy $\mathrm{x}$-ray absorptiometry. Biomed Res Int 2014; 2014. doi:10.1155/2014/253067.

25 Ayala JE, Samuel VT, Morton GJ, Obici S, Croniger CM, Shulman GI et al. Standard operating procedures for describing and performing metabolic tests of glucose homeostasis in mice. Dis Model Mech 2010; 3: 525-534.

26 Marini C, Salani B, Massollo M, Amaro A, Esposito AI, Orengo AM et al. Direct inhibition of hexokinase activity by metformin at least partially impairs glucose metabolism and tumor growth in experimental breast cancer. Cell Cycle 2013; 12: 3490-3499.

27 Marini C, Bianchi G, Buschiazzo A, Ravera S, Martella R, Bottoni G et al. Divergent targets of glycolysis and oxidative phosphorylation result in additive effects of metformin and starvation in colon and breast cancer. Sci Rep 2016; 6: 19569.

28 Bianchi G, Martella R, Ravera S, Marini C, Capitanio S, Orengo A et al. Fasting induces 
anti-Warburg effect that increases respiration but reduces ATP-synthesis to promote apoptosis in colon cancer models. Oncotarget 2015; 6: 11806-19.

29 Garbarino S, Vivaldi V, Delbary F, Caviglia G, Piana M, Marini C et al. A new compartmental method for the analysis of liver FDG kinetics in small animal models. EJNMMI Res 2015; 5: 35.

30 Patlak CS, Blasberg RG, Fenstermacher JD. Graphical Evaluation of Blood-to-Brain Transfer Constants from Multiple-Time Uptake Data. 1983; : 1-7.

31 Amri EZ, Bertrand B, Ailhaud G, Grimaldi P. Regulation of adipose cell differentiation. I. Fatty acids are inducers of the aP2 gene expression. J Lipid Res 1991; 32: 1449-56.

32 Tso AW, Xu A, Chow WS, Lam KS. Adipose tissue and the metabolic syndrome: focusing on adiponectin and several novel adipokines. Biomark Med 2008; 2: 239-52.

33 Weisberg SP, McCann D, Desai M, Rosenbaum M, Leibel RL, Ferrante AW. Obesity is associated with macrophage accumulation in adipose tissue. J Clin Invest 2003; 112: 17961808.

34 Costa D, Principi E, Lazzarini E, Descalzi F, Cancedda R, Castagnola P et al. LCN2 overexpression in bone enhances the hematopoietic compartment via modulation of the bone marrow microenvironment. J Cell Physiol 2017; : 1-24.

35 Shoelson SE, Herrero L, Naaz A. Obesity, Inflammation, and Insulin Resistance. Gastroenterology 2007; 132: 2169-2180.

36 Myers MG, Cowley MA, Unzberg H. Mechanisms of Leptin Action and Leptin Resistance. Annu Rev Physiol 2008; 70: 537-56.

37 Brennan AM, Mantzoros CS. Drug Insight: the role of leptin in human physiology and pathophysiology--emerging clinical applications. Nat Clin Pr Endocrinol Metab 2006; 2: 318-327.

38 Campfield LA, Smith FJ, Guisez Y, Devos R, Burn P. Recombinant mouse OB protein: evidence for a peripheral signal linking adiposity and central neural networks [see comments]. Science (80- ) 1995; 269: 546-549.

39 Steinberg GR, Smith AC, Wormald S, Malenfant P, Collier C, Dyck DJ. Endurance training partially reverses dietary-induced leptin resistance in rodent skeletal muscle. Am J Physiol Endocrinol Metab 2004; 286: E57-63.

40 Hotamisligil GS. Inflammation and metabolic disorders. Nature 2006; 444: 860-867.

41 Wang Y, Lam KSL, Kraegen EW, Sweeney G, Zhang J, Tso AWK et al. Lipocalin-2 is an inflammatory marker closely associated with obesity, insulin resistance, and hyperglycemia in humans. Clin Chem 2007; 53: 34-41.

42 Yan QW, Yang Q, Mody N, Graham TE, Hsu CH, Xu Z et al. The adipokine lipocalin 2 is regulated by obesity and promotes insulin resistance. Diabetes 2007; 56: 2533-2540.

43 Guo H, Jin D, Zhang Y, Wright W, Bazuine M, Brockman DA et al. Lipocalin-2 deficiency impairs thermogenesis and potentiates diet-induced insulin resistance in mice. Diabetes 2010; 59: 1376-1385.

44 Clemens TL, Karsenty G. The osteoblast: An insulin target cell controlling glucose 
homeostasis. J Bone Miner Res 2011; 26: 677-680. 


\section{FIGURE LEGENDS}

Figure 1. Mice weight and metabolism. In all the experiments, $\mathrm{N} \geq 6 \mathrm{Wt}$ and $\mathrm{LCN} 2-\mathrm{Tg}(\mathrm{Tg}) 18$ month old mice were analysed, unless otherwise specified.

(A) Analysis of the weight variance in relationship to the length of the animals. (B) Analysis and histogram of the average of the body mass index (BMI). (C) Analysis of the variance of the weight of the visceral fat in relationship to the total weight of the animals ( $n=3)$. (D) Representative pictures that show the interspersed variability in the amount of visceral fat between $\mathrm{Tg}$ mice in relationship to the one observed in Wt mice. (E) Adipose tissue real-time PCR for LCN2 mRNA expression. (F) Metabolic cages analysis for food and water intake.

Results are means \pm SEM. Data were analysed with student t-test. ${ }^{*} \mathrm{p}<0.05, * * \mathrm{p}<0.01, * * * \mathrm{p}<$ 0.001 .

\section{Figure 2. Physiology and inflammatory status of the visceral adipose tissue.}

In all the experiments, $\mathrm{N} \geq 6 \mathrm{Wt}$ and $\mathrm{LCN} 2-\mathrm{Tg}$ (Tg) 18 month old mice were analysed, unless otherwise specified. (A) Top panels shows representative pictures of visceral adipose tissue in Wt and $\mathrm{Tg}$ mice with body weight $<20 \mathrm{~g}(\mathrm{Tg} 1)$ and $>20 \mathrm{~g}(\mathrm{Tg} 2)$; bottom panels show correspondent representative pictures of Hematoxilin Eosin (H\&E) staining of the visceral adipose tissue. (B) Adipocytes number per area in $\mathrm{H} \& \mathrm{E}$ of $\mathrm{Wt}$ and $\mathrm{Tg}$ mice $<20 \mathrm{~g}$ and $>20 \mathrm{~g}$ of weight. (C) Adipocyte area expressed in $\mathrm{m}^{2}$ of LCN2-Tg mice with body weight $<20 \mathrm{~g}$ and $>20 \mathrm{~g}$. (D) Adipose tissue realtime PCR analysis for PPAR $\gamma$ and SRBF1. Y-axis represents the mRNA fold increases of each gene versus Wt. (E) Adipose tissue Real-time PCR analysis for FBP4, Leptin, TNF $\alpha$ and IL-6. Y-axis represents the mRNA fold increases of each gene versus Wt. (F) FACS analyses for fat resident macrophages (MФ) subpopulations percentage.

Results are means \pm SEM. ${ }^{*} \mathrm{p}<0.05,{ }^{*} \mathrm{p}<0.01,{ }^{* *} \mathrm{p}<0.001, * * * * \mathrm{p}<0.0001$

\section{Figure 3: Glucose tolerance test (GTT).}


$\mathrm{N} \geq 6 \mathrm{Wt}$ and LCN2-Tg (Tg) 18 month old mice were starved for 8 hours and challenged with glucose $(1,5 \mathrm{mg} / \mathrm{g})$ by intraperitoneal injection. Glucose concentrations were monitored at Time 0 and at different time points after the glucose intraperitoneal injection. Results are means $\pm \mathrm{SEM}$. $* \mathrm{p}<0.05$.

Figure 4: Micro-PET analysis. $\mathrm{N} \geq 6 \mathrm{Wt}$ and LCN2-Tg (Tg) 18 month old mice were studied twice in random sequence. (A) Blood glucose concentrations before micro-PET analysis. (B) FDG detection by radiographic image in whole body. Arrows indicate brown fat FDG disposal in Wt and Tg mice. Fat glucose metabolic rate (MRGlu) in Whole body (C), muscle (D), and brown adipose tissue (E). Phosphorilation (kmf) (F) and dephosphorilation (G) rate (kfm) of FDG in hepatocytes. Results are means \pm SEM. ${ }^{*} \mathrm{p}<0.05$

Figure 5: Serum analysis. $\mathrm{N} \geq 6 \mathrm{Wt}$ and $\mathrm{LCN} 2-\mathrm{Tg}(\mathrm{Tg}) 18$ month old mice were sacrificed and cardiac blood samples collected. Histograms of the amount of circulating: total cholesterol and triglycerides (A), creatinine (B), biliary acids (C), total bilirubin (D), urea (E) and aspartate aminotransferase (AST) and alanine aminotransferase (ALT) levels (F).

Results are mean \pm SEM. ${ }^{*} \mathrm{p}<0.05$ 
$A$

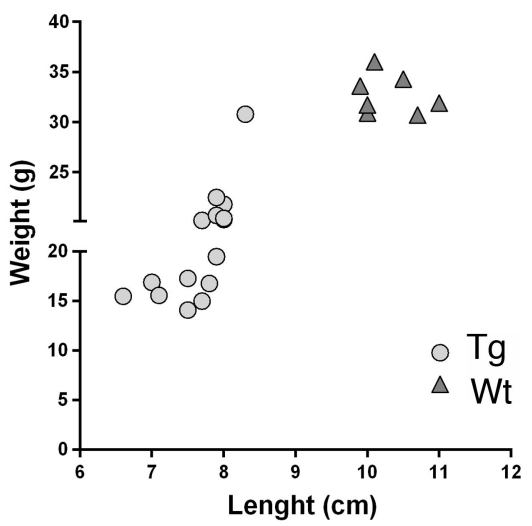

C
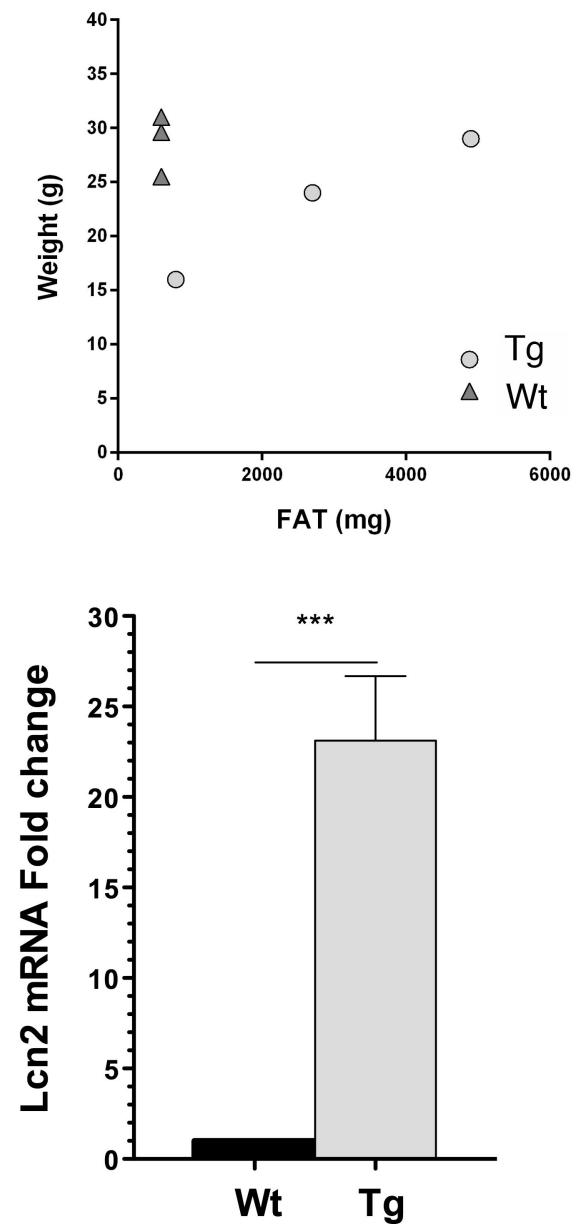

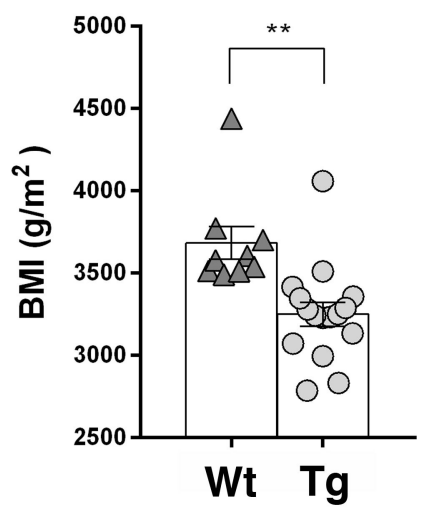

D

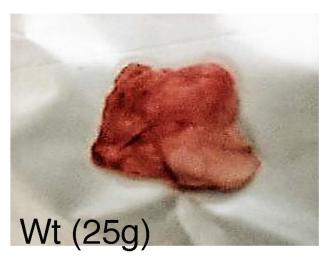

$\operatorname{Tg}(16 g)$

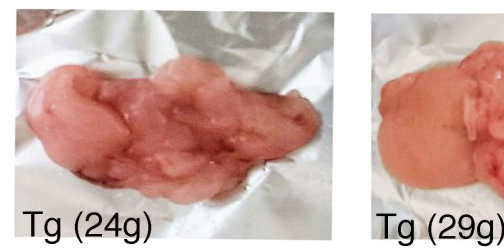

F

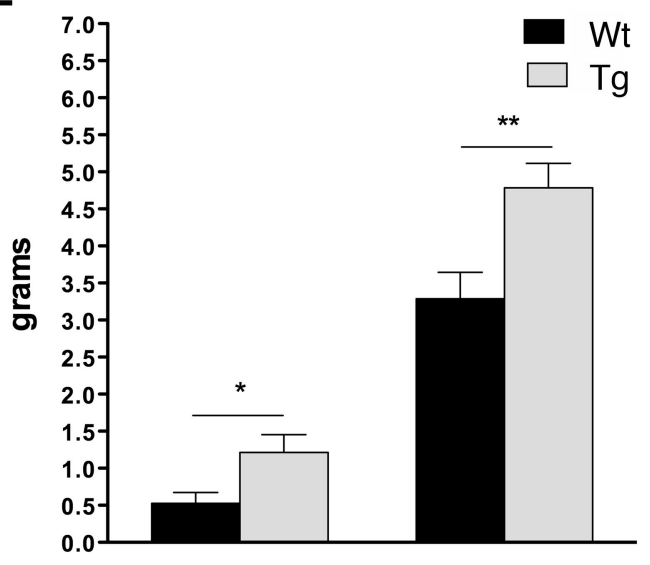

Food 


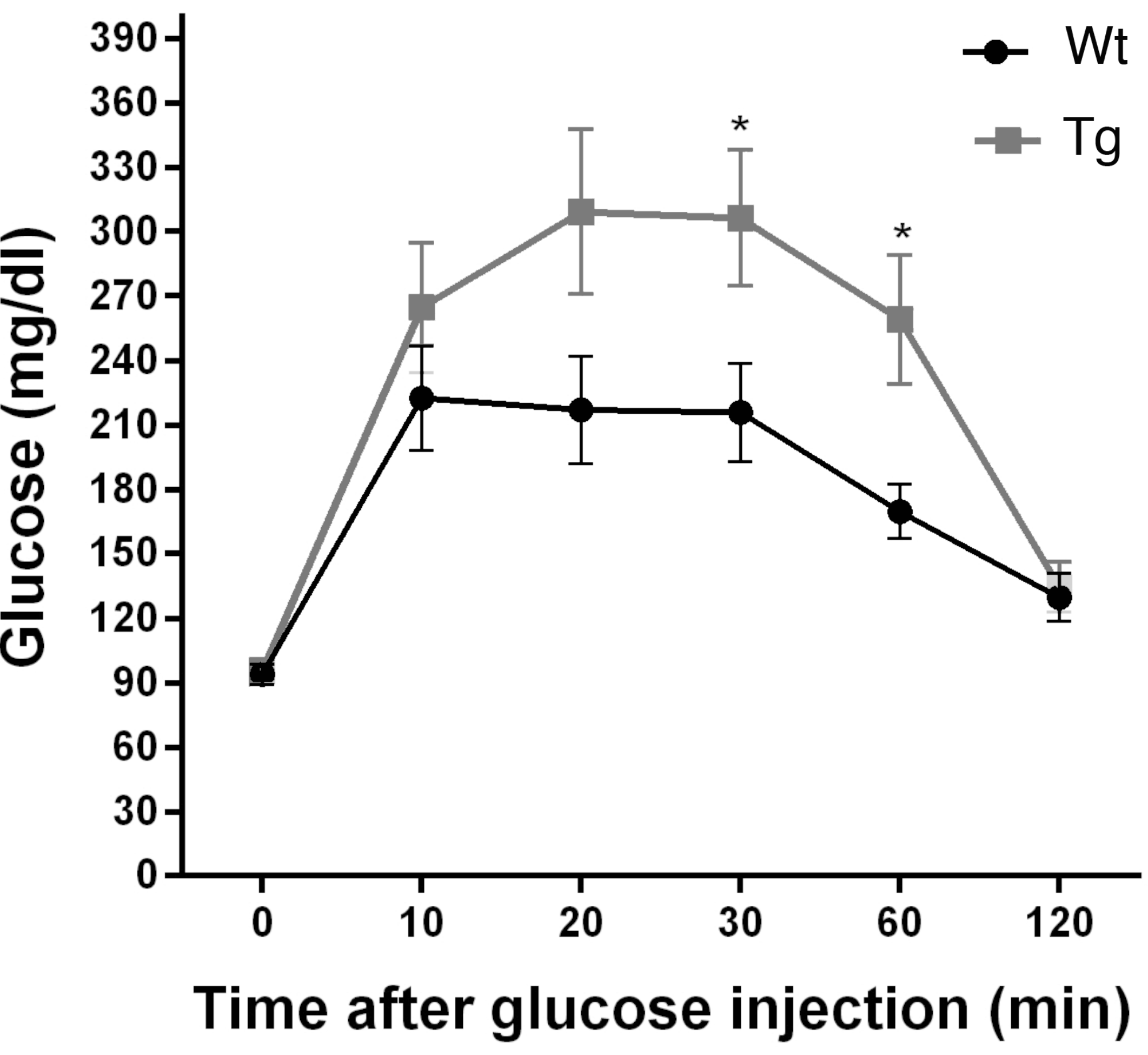




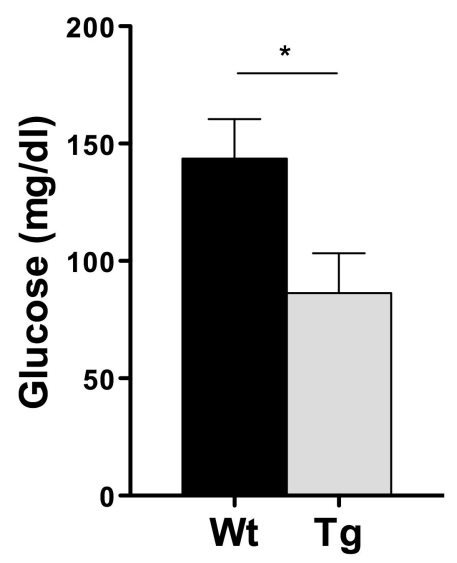

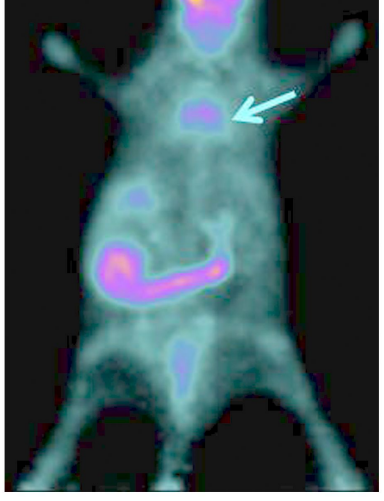

Wt
$\mathrm{Tg}$

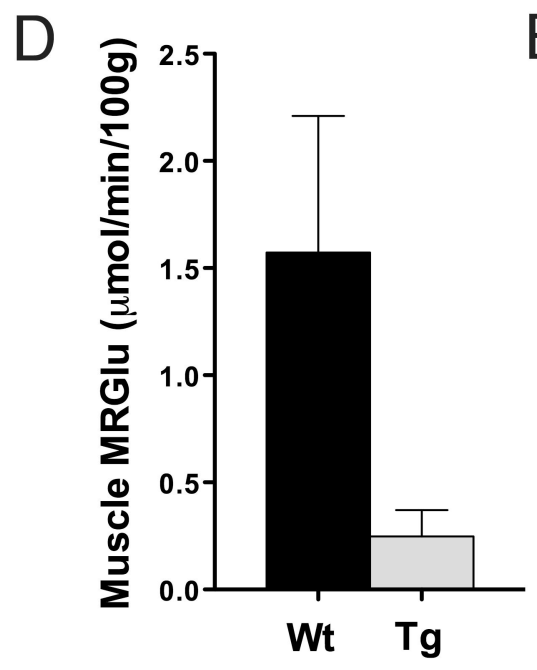

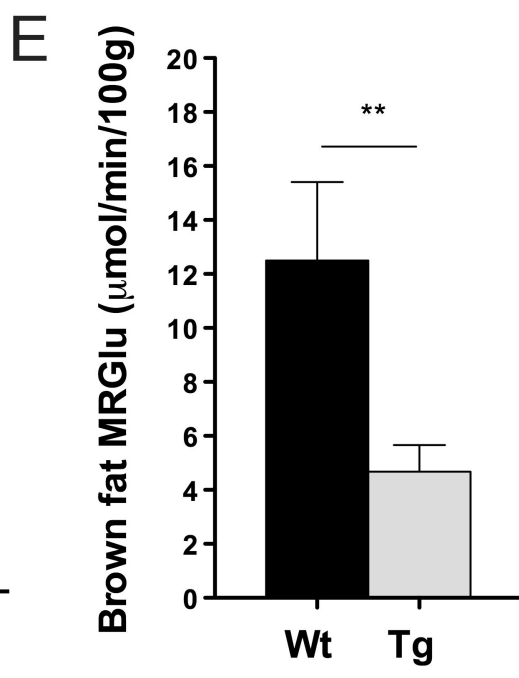

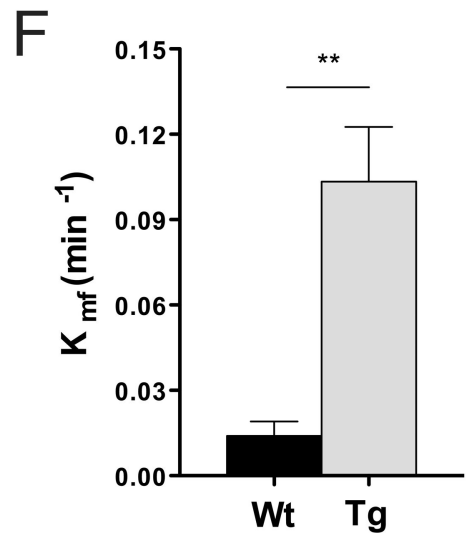

G

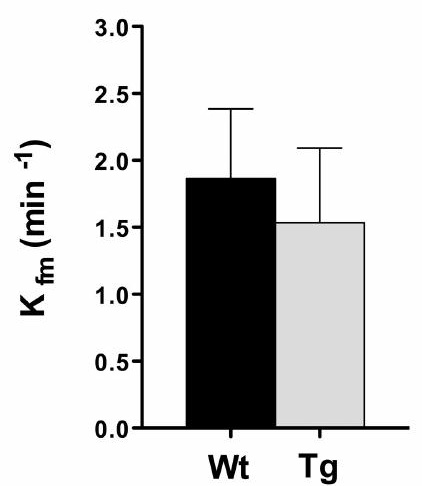


A
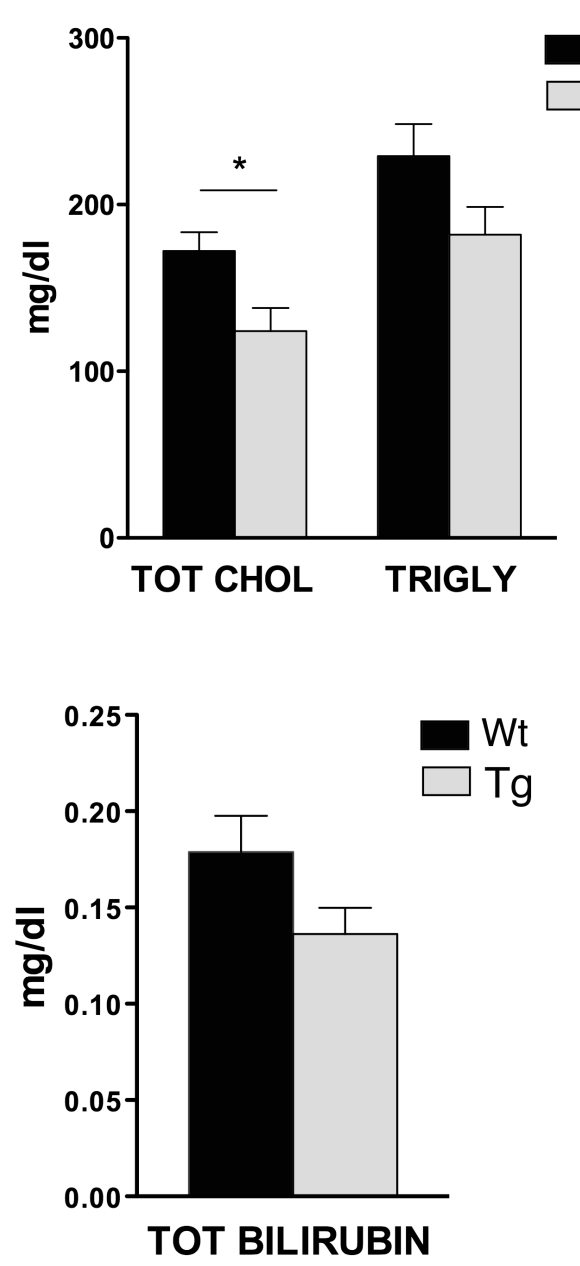

B

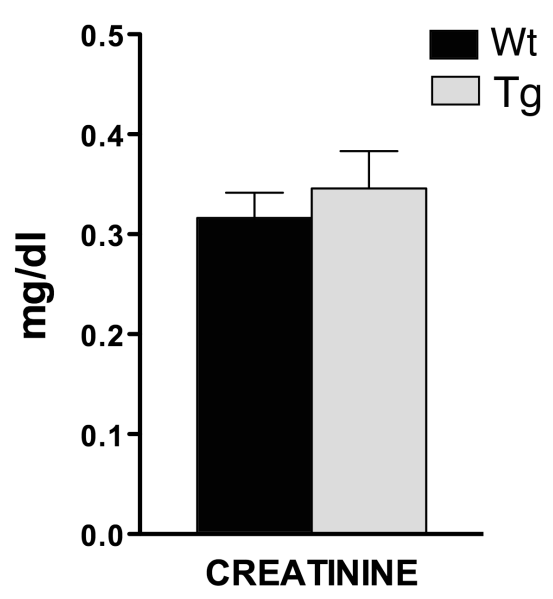

E

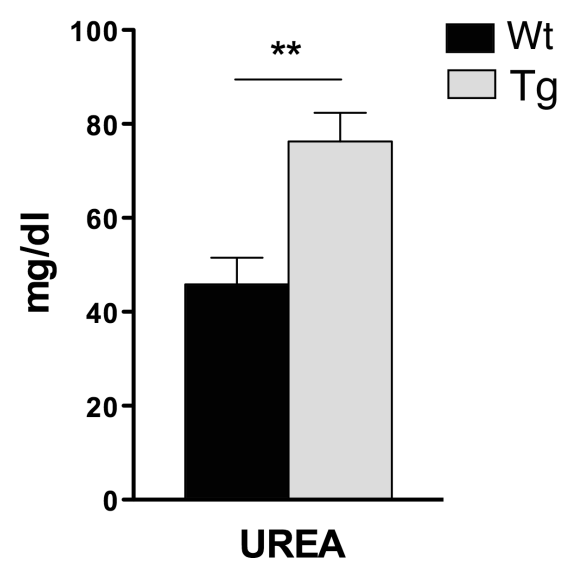

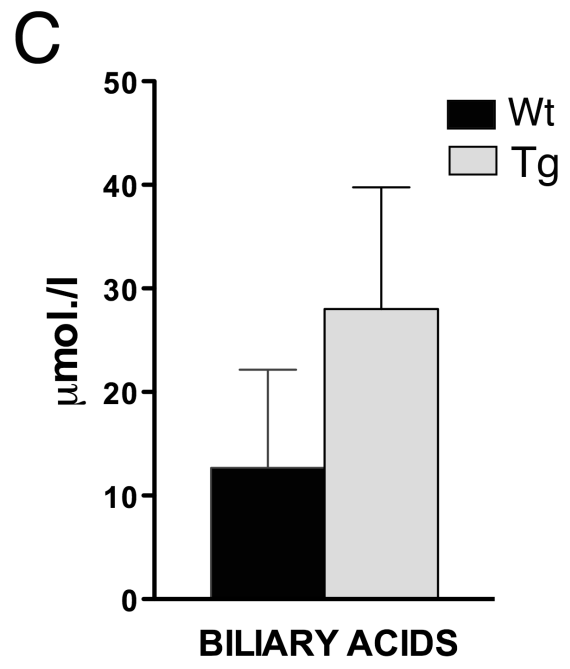

F

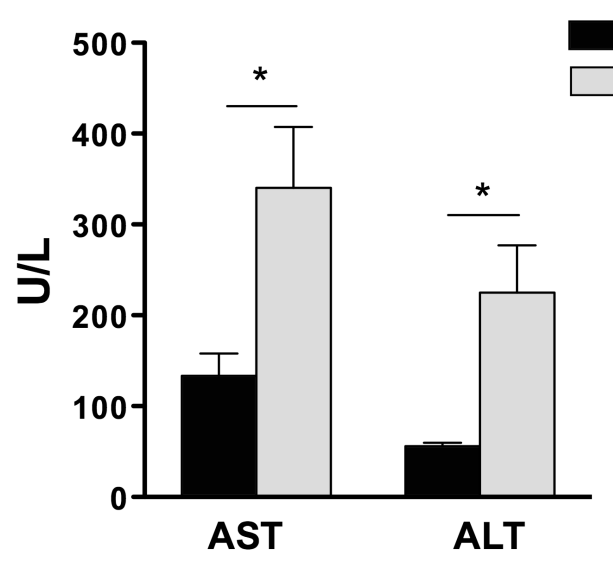




\begin{tabular}{|l|lc|}
\hline \multicolumn{1}{|c|}{ GENE } & \multicolumn{3}{|c|}{ PRIMER SEQUENCES } \\
\hline Interleukin 6 (IL-6) & 5'-GTCCGGAGAGGAGACTTCAC-3' & Forward \\
& 5'-CTGCAAGTGCATCATCGTTGT-3 & Reverse \\
\hline Fat acid binding protein (FBP4) & 5'-TGAGCTGAAGTCGCTTCCTG-3' & Forward \\
& 5'-TCTGAGAGCATCAAAGGAGCC & Reverse \\
\hline \multirow{2}{*}{ Leptin } & 5'-CATTTCACACACGTCGGTATCC & Forward \\
\hline Tumor necrosis factor alpha (TNF $\alpha)$ & 5'-CACATTTTGGGAAGGCAGGC-3' & Reverse \\
\hline Peroxisome proliferator- & 5'-TGTCCCTTTCACTCACTGGC-3' & Forward \\
activatedreceptor gamma (PPARY) & 5'-TTGAGTTTGGCTGTGAAGTTCAA' & Forward \\
\hline Adipocyte determination and & 5'-CAGCAGGTTGTCTTGGATGT-3' & Reverse \\
\hline differentiation factor 1 (SRBF1) & 5'-ATGTGTGCAGGTGACAGTCC-3' & Forward \\
\hline Lipocalin2 (LCN2) & 5'-TAGGCACTTGGTACACGCTG-3' & Reverse \\
\hline
\end{tabular}

